\title{
An integral treatment for heat and mass transfer along a vertical wall by natural convection in a porous media
}

\author{
B. B. Singh \\ Department of Mathematics, \\ Dr. Babasaheb Ambedkar Technological University, Lonere-402103, \\ Dist. Raigad (M.S.), India
}

\begin{abstract}
This paper deals with the free convective heat and mass transfer along a vertical wall embedded in a fluid saturated porous medium by using an integral method of the Von-Karman type in the presence of temperature and concentration gradients. Mathematical expressions for the local Nusselt number and local Sherwood number have been derived in terms of boundary layer thickness ratio. The governing parameters for the flow-field are buoyancy ratio $(\mathrm{N})$ and Lewis number (Le). The numerical values of the local Nusselt number and local Sherwood number have been computed for a wide range of values of $\mathrm{N}$ and Le. The variations of local Nusselt number and local Sherwood number with $\mathrm{N}$ have also been studied with the help of graphs for the different values of Le. Similarly, the variations of local Nusselt number and local Sherwood number with Le have been studied for different values of $\mathrm{N}$ with the help of graphs. It has been found that the local Nusselt number increases as $\mathrm{N}$ increases for the decreasing value of Le, whereas the local Sherwood number increases as $\mathrm{N}$ increases for the increasing values of Le. The local Nusselt number and the local Sherwood number increase as Le increases for increasing values of $\mathrm{N}$. The numerical values of the thermal boundary layer and concentration boundary layer thicknesses have also been computed for the flow-field. It has been found that the results obtained by the integral method are in good agreement with those obtained by Bejan and Khair [Heat and Mass Transfer by Natural Convection in a Porous medium, Int. J. Heat Mass Transfer, 28, pp. 909-918, 1985].

Keywords: natural convection, porous media, heat and mass transfer.
\end{abstract}




\section{Introduction}

The natural convection flows arising out of the combined buoyancies due to thermal and mass diffusion in a porous medium are of importance because of the fundamental nature of the problem and broad range of their applications pertaining to manufacturing and process industries such as geothermal systems, fibre and granular insulation, storage of nuclear waste materials, usage of porous conical bearings in lubrication technology, chemical catalytic reactors, dispersion of chemical contaminant through water saturated soil , natural gas storage tanks , etc.

On account of the afore-mentioned applications, Bejan and Khair [1] used the Darcy's law to study the features of the free convection boundary layer flow driven by temperature and concentration gradients. Recently, Lai and Kulacki [2] have re-examined the free convection boundary layer along a vertical wall with constant heat and mass flux including the effect of wall injection. The heat and mass transfer by natural convection near a vertical wall in a porous medium under boundary layer approximations has been studied by Nakayama and Hossain [3] and Singh and Queeny [4]. A further review of coupled heat and mass transfer by natural convection in porous medium is given by Nield and Bejan [5].

The objective of the present paper is to apply integral method to analyze free convection problem along a vertical wall in the presence of temperature and concentration gradients. A comparison of the numerical values of the local Nusselt and local Sherwood numbers obtained by the integral method has been done with those obtained by Bejan and Khair [1] for different values of the buoyancy ratio.

It has been found that the results obtained by the present method are in good agreement with those obtained by Bejan and Khair.

\section{Mathematical analysis}

We consider a two-dimensional laminar flow over a vertical flat plate in a porous medium embedded in a Darcian fluid. The co-ordinate system and the physical model are shown in figure 1. In the mathematical formulation of the problem, we note the following conventional assumptions:

i) the physical properties are considered to be constant, except for the density term that is associated with the body force;

ii) flow is sufficiently slow so that the convecting fluid and the porous matrix are in local thermodynamic equilibrium;

iii) Darcy's law, the Boussinesq and boundary layer approximations have been employed.

With these assumptions, the governing equations are given by 


$$
\begin{gathered}
\frac{\partial u}{\partial x}+\frac{\partial v}{\partial y}=0 \\
u=\frac{g K}{v}\left(\beta _ { T } \left(T-T_{\infty}+\beta_{C}\left(C-C_{\infty}\right)\right.\right. \\
u \frac{\partial T}{\partial x}+v \frac{\partial T}{\partial y}=\alpha \frac{\partial^{2} T}{\partial y^{2}} \\
u \frac{\partial C}{\partial x}+v \frac{\partial C}{\partial y}=D \frac{\partial^{2} C}{\partial y^{2}}
\end{gathered}
$$

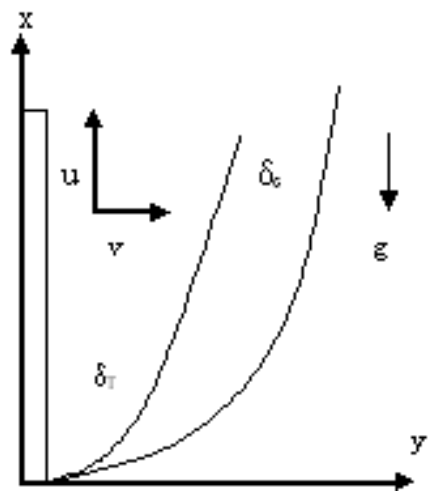

Figure 1: Physical model and co- Figure 2: Heat transfer coefficient ordinate system.

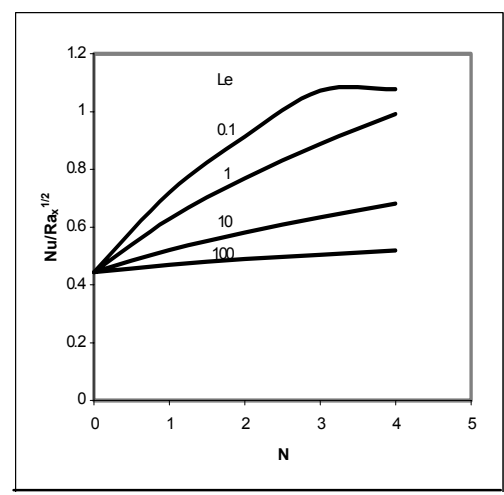

as a function of buoyancy ratio.

The symbols have got their meanings as mentioned in the Nomenclature.

The boundary conditions at the wall are

$$
\mathrm{y}=0: \mathrm{v}=0, \mathrm{~T}=\mathrm{T}_{\mathrm{w}}, \mathrm{C}=\mathrm{C}_{\mathrm{w}}
$$

and at infinity are

$$
\mathrm{y} \rightarrow \infty ; \mathrm{u}=0, \mathrm{~T} \rightarrow \mathrm{T}_{\infty}, \mathrm{C} \rightarrow \mathrm{C}_{\infty}
$$

\section{Integral method}

The boundary layer equations (2)-(4) along with boundary conditions (5) and (6) have been solved by using integral method. The partial differential equations get converted into the ordinary differential equations by making use of the following transformations: 
146 Computational Methods in Multiphase Flow IV

$$
\begin{gathered}
\eta=\frac{y}{x}\left(R a_{x}\right)^{1 / 2} \\
\psi=\alpha\left(R a_{x}\right)^{1 / 2} f(\eta) \\
\theta=\frac{T-T_{w}}{T_{W}-T_{\infty}} \\
\phi=\frac{C-C_{w}}{C_{w}-C_{\infty}}
\end{gathered}
$$

where $R a_{x}=\frac{g \beta_{T} K x\left(T_{w}-T_{\infty}\right)}{\alpha v}$ is the modified local Rayleigh number, $\psi$ is the stream function.

After transformation the resulting equations become

$$
\begin{gathered}
f^{\prime \prime}(\eta)-\theta^{\prime}(\eta)-N \phi^{\prime}(\eta)=0 \\
\theta^{\prime \prime}(\eta)+1 / 2 f(\eta) \theta^{\prime}(\eta)=0 \\
\phi^{\prime \prime}(\eta)+\left(\text { Le/2) } f(\eta) \phi^{\prime}(\eta)=0\right.
\end{gathered}
$$

with boundary conditions

$$
\begin{gathered}
f(0)=0, \theta(0)=\phi(0)=1 \\
f^{\prime}(\infty)=\theta(\infty)=\phi(\infty)=0
\end{gathered}
$$

where primes denote the differentiation with respect to ' $\eta$ ', $\eta \in[0, \infty)$. Here, $\mathrm{f}^{\prime}(\eta)$ is non-dimensional velocity related to the stream function $\psi(x, y)$.

In the above equations (11) - (13), $\mathrm{N}$ is the buoyancy ratio defined by

$$
N=\frac{\beta_{C}\left(C_{w}-C_{\infty}\right)}{\beta_{T}\left(T_{w}-T_{\infty}\right)}
$$

and Le is the Lewis number defined by

$$
\text { Le }=\alpha / D
$$

From (12) and (13), we get

$$
\begin{aligned}
& -\theta^{\prime}(0)=\frac{1}{2} \int_{0}^{\infty} f^{\prime} \phi d \eta \\
& -\theta^{\prime}(0)=\frac{L e}{2} \int_{0}^{\infty} f^{\prime} \phi d \eta
\end{aligned}
$$


The infinity is boundary layer thickness for temperature and concentration. We now assume the exponential temperature and concentration profiles as follows:

$$
\begin{gathered}
\theta(\eta)=\exp \left(-\eta / \delta_{\mathrm{T}}\right) \\
\phi(\eta)=\exp \left(-\xi \eta / \delta_{\mathrm{T}}\right)
\end{gathered}
$$

Here $\delta_{\mathrm{T}}$ is arbitrary scale for the thermal boundary layer thickness whereas $\xi$ is its ratio to the concentration boundary thickness $\delta_{\mathrm{C}}$. With the help of above profiles, and using equation (11), the equations (18) and (19) can be obtained in two distinct expressions as

$$
\begin{array}{r}
\frac{1}{\delta_{T^{2}}}=\frac{\xi+1+2 N}{4(\xi+1)} \\
\frac{1}{\delta_{T^{2}}}=\left\{\frac{2 \xi+N(\xi+1)}{4 \xi^{2}(\xi+1)} L e\right\}
\end{array}
$$

The above two equations (22) and (23) can be combined together to give the following cubic equation for determining the boundary layer thickness ratio $\xi$ as

$$
\xi^{3}+(1+2 \mathrm{~N}) \xi^{2}-[(2+\mathrm{N}) \mathrm{Le}] \xi-\mathrm{N} \mathrm{Le}=0
$$

As $\xi$ is determined by using the computer programming like MATLAB from the equation (24), the local Nusselt and Sherwood numbers which are of our main interest in terms of heat and mass transfer respectively, are given as

$$
\frac{N u}{\left(R a_{x}\right)^{1 / 2}}=0.5\left(\frac{\xi+1+2 N}{\xi+1}\right)^{1 / 2}
$$

and

$$
\frac{S h}{\left(R a_{x}\right)^{1 / 2}}=0.5 \xi\left(\frac{\xi+1+2 N}{\xi+1}\right)^{1 / 2}
$$

The accuracy acquired in the above approximations may be examined by comparing the heat and mass transfer results against those obtained by Bejan and Khair [1]. It is not unusual to have an error of $5 \%$ or more, depending on the assumed profile. However, the situation can be remedied by adjusting the multiplicative constant, namely, replacing 0.5 by 0.444 . Thus, the following approximate formulae are proposed: 


$$
\begin{aligned}
& \frac{N u}{\left(R a_{x}\right)^{1 / 2}}=0.444\left(\frac{\xi+1+2 N}{\xi+1}\right)^{1 / 2} \\
& \frac{S h}{\left(R a_{x}\right)^{1 / 2}}=0.5 \xi\left(\frac{\xi+1+2 N}{\xi+1}\right)^{1 / 2}
\end{aligned}
$$

\section{Results and discussions}

The formulae (27) and (28) give the values of the local Nusselt number ( $\mathrm{Nu}$ ) and Sherwood number ( $\mathrm{Sh}$ ) as 0.444 for $\mathrm{N}=0$ and $\mathrm{Le}=1$. These values are the same as obtained by Bejan and Khair [1]. The above assertion is clear from table 1 . We have done calculations for a wide range of the parameters $\mathrm{N}$ (buoyancy ratio) and Le (Lewis number) in order to understand their influence on the combined heat and mass transfer along a vertical wall due to free convection. These values have been given in table 1 . From the table, it is evident that the values of local $\mathrm{Nu}$ and $\mathrm{Sh}$ obtained by the integral method for different values of Le are in excellent agreement with those obtained by Bejan and Khair who obtained the corresponding values by the similarity solution technique.

From the table, it is clear that the thermal boundary layer thickness $\delta_{\mathrm{T}}$ shows an increasing trend for $\mathrm{N}=1,4$ for the increasing values of the Lewis number Le. On the contrary, the concentration boundary layer thickness $\delta_{\mathrm{C}}$ shows a decreasing trend for $\mathrm{N}=0,1,4$ for the increasing values of Le. From the table, it is obvious that the Lewis number has more pronounced effect on the concentration field than it has on temperature field. From the table, it is further evident that the magnitudes of the thermal boundary layer and concentration boundary layer thicknesses are equal for $\mathrm{N}=0, \mathrm{Le}=1 ; \mathrm{N}=1, \mathrm{Le}=1$ and $\mathrm{N}=4$, $\mathrm{Le}=1$.

The local Nusselt number has been plotted in figure 2 as a function of buoyancy ratio for various values of Lewis number $(\mathrm{Le}=0.1,1,10,100)$. It is found that the rate of heat transfer decreases with increasing Lewis number for $\mathrm{N}$ $>0$. Similarly the local Sherwood number has been plotted in figure 3 against the buoyancy ratio $\mathrm{N}$ for various values of the Lewis number $(\mathrm{Le}=1,10,50,100)$. It is found that the rate of mass transfer increases with increasing Lewis number for all $\mathrm{N}$.

The local Nusselt number has been plotted in figure 4 as a function of Lewis number for various values of buoyancy ratio $\mathrm{N}=0,2$ and 4 . It is found that the local Nusselt number decreases with increasing Lewis number for $\mathrm{N}>0$. Similarly the local Sherwood number is plotted in figure 5 as a function of Lewis number for various values of buoyancy ratio $\mathrm{N}=0,1$ and 4 . It is found that the local Sherwood number increases with increasing Lewis number for all N. From figures 4 and 5, also it is evident that the values of local Nusselt and local Sherwood numbers in the present case are in excellent agreement with those obtained by Bejan and Khair. 
Table 1: $\quad$ Comparison of Local Nusselt and Sherwood numbers.

\begin{tabular}{|cccc|cccc|}
\hline & \multicolumn{3}{c|}{$\mathrm{Nu} /\left(\mathrm{Ra}_{\mathrm{x}}\right)^{1 / 2}$} & \multicolumn{5}{c|}{$\mathrm{Sh} /\left(\mathrm{Ra}_{\mathrm{x}}\right)^{1 / 2}$} \\
\hline $\mathrm{N}$ & Le & Numerical & Present & Numerical & Present & $\delta_{\mathrm{T}}$ & $\delta_{\mathrm{C}}$ \\
& 1 & 0.444 & 0.444 & 0.444 & 0.444 & 2 & 1 \\
& 2 & 0.444 & 0.444 & 0.683 & 0.693 & 2 & 1.2807 \\
& 4 & 0.444 & 0.444 & 1.019 & 1.053 & 2 & 0.8430 \\
& 6 & 0.444 & 0.444 & 1.275 & 1.332 & 2 & 0.6666 \\
& 8 & 0.444 & 0.444 & 1.491 & 1.568 & 1.9999 & 0.5663 \\
& 10 & 0.444 & 0.444 & 1.680 & 1.776 & 2 & 0.5 \\
& 100 & 0.444 & 0.444 & 5.544 & 6.061 & 2 & 0.1455 \\
\hline 1 & 1 & 0.628 & 0.628 & 0.628 & 0.628 & 1.4142 & 1.4142 \\
& 2 & 0.593 & 0.591 & 0.930 & 0.937 & 1.5015 & 0.9478 \\
& 4 & 0.559 & 0.557 & 1.358 & 1.383 & 1.5935 & 0.6418 \\
& 6 & 0.541 & 0.539 & 1.685 & 1.728 & 1.6459 & 0.5138 \\
& 8 & 0.529 & 0.528 & 1.960 & 2.019 & 1.6806 & 0.4395 \\
& 10 & 0.521 & 0.520 & 2.202 & 2.276 & 1.7074 & 0.3901 \\
& 100 & 0.470 & 0.4692 & 7.139 & 7.539 & 1.8733 & 0.1166 \\
\hline 4 & 1 & 0.992 & 0.992 & 0.992 & 0.992 & 0.8944 & 0.8944 \\
& 2 & 0.899 & 0.896 & 1.431 & 1.436 & 0.9905 & 0.6180 \\
& 4 & 0.793 & 0.797 & 2.055 & 2.017 & 1.1138 & 0.4284 \\
& 6 & 0.742 & 0.743 & 2.533 & 2.562 & 1.1951 & 0.3464 \\
& 8 & 0.707 & 0.707 & 2.936 & 2.976 & 1.2543 & 0.2979 \\
& 10 & 0.681 & 0.681 & 3.290 & 3.341 & 1.3030 & 0.2657 \\
& 100 & 0.521 & 0.519 & 10.521 & 10.792 & 1.6630 & 0.08 \\
\hline
\end{tabular}

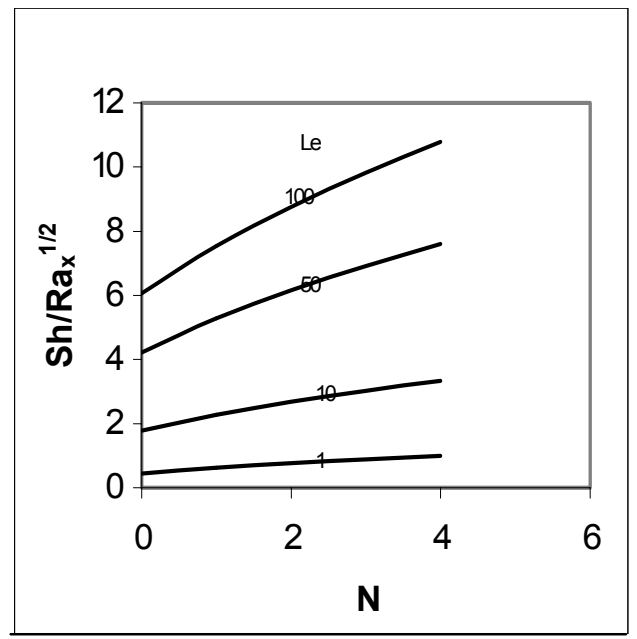

Figure 3: $\quad$ Mass transfer coefficient as a function of buoyancy ratio. 


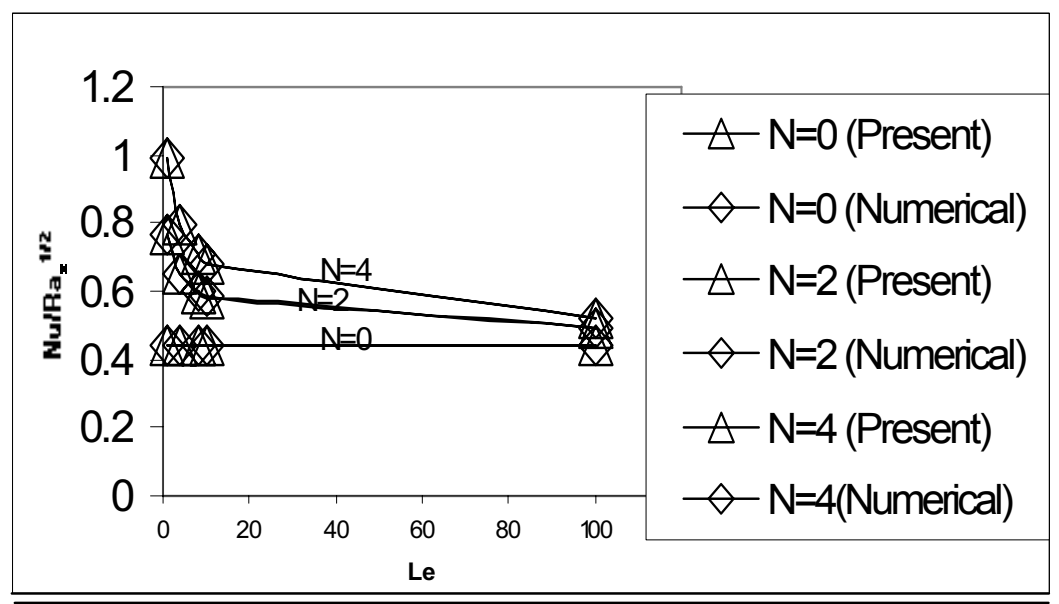

Figure 4: Heat transfer results.

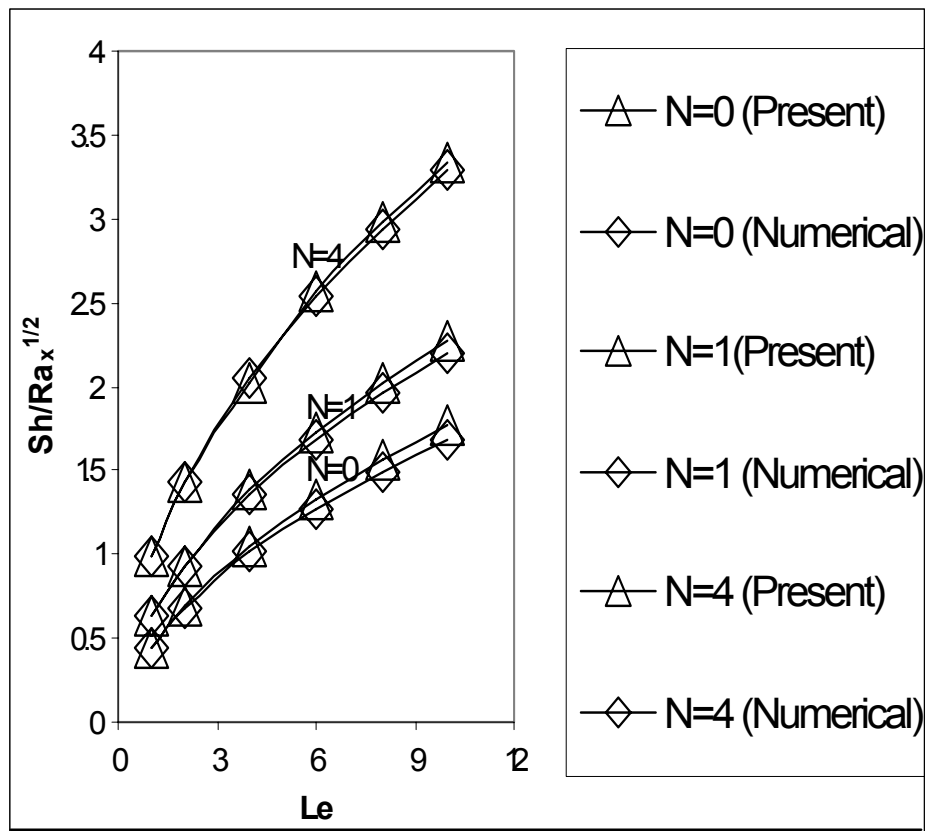

Figure 5: $\quad$ Mass transfer results.

\section{Concluding remarks}

This paper deals with the free convective heat and mass transfer along a vertical wall embedded in a fluid saturated porous medium. The heat and mass transfer coefficients obtained in the present study by the integral method agree very well 
with those obtained by Bejan and Khair. In the present analysis, the results have been presented in such a way that any practicing engineer can easily obtain the physical characteristic of the problem for arbitrary values of the buoyancy ratio and Lewis number. The advantage of this method is that it also provides with great freedom the approximate solutions to non-linear problems. The further advantage of this method is that the results are obtained with more ease as compared to Bejan and Khair.

\section{Nomenclature}

$\begin{array}{ll}\mathrm{N} & \text { buoyancy ratio } \\ \mathrm{T} & \text { temperature } \\ \mathrm{C} & \text { concentration } \\ \mathrm{D} & \text { mass diffusivity of porous } \\ & \text { medium } \\ \mathrm{f} & \text { dimensionless stream function } \\ \mathrm{g} & \text { gravitational acceleration } \\ \mathrm{h} & \text { local heat transfer coefficient } \\ \mathrm{k} & \text { thermal conductivity } \\ \mathrm{K} & \text { permeability } \\ \mathrm{Le} & \text { Lewis number } \\ \mathrm{Nu} & \text { local Nusselt number } \\ \mathrm{Sh} & \text { local Sherwood number } \\ \mathrm{Ra} & \text { modified Rayleigh number } \\ \mathrm{u} & \text { Darcy's velocity in } \mathrm{x}-\text { direction } \\ \mathrm{V} & \text { Darcy's velocity in } \mathrm{y}-\text { direction } \\ \mathrm{X}, \mathrm{y} & \text { cartesian co-ordinate }\end{array}$

\section{Greek Symbols}

$\alpha$ thermal diffusivity of porous medium

$\eta \quad$ similarity variable

$\beta_{\mathrm{T}} \quad$ coefficient of thermal expansion

$\beta_{\mathrm{C}}$ coefficient of concentration expansion

$\delta_{\mathrm{T}} \quad$ arbitrary length scale for thermal boundary layer

$\delta_{\mathrm{C}}$ arbitrary length scale for concentration boundary layer

$\psi \quad$ stream function

$\phi$ dimensionless concentration

$\xi \quad$ boundary layer thickness ratio

$\checkmark$ kinematic viscosity

\section{Subscripts}

$\infty$ condition at the infinity

w condition at the wall

\section{References}

[1] Bejan, A. and Khair, K.R., Heat and Mass Transfer by Natural Convection in a Porous medium, Int. J. Heat Mass Transfer, 28, pp. 909-918, 1985.

[2] Lai, F.C. and Kulacki, Coupled Heat and Mass Transfer by Natural Convection from Vertical Surface in Porous Media, Int. J. Heat Mass Transfer, 34, pp. 1189-1194, 1991.

[3] Nakayama, A. and Hossain, M. A., An Integral Treatment for Combined Heat and Mass Transfer by Natural Convection in a Porous Media, Int. J. Heat Mass Transfer, 38, pp. 761-765, 1995.

[4] Singh, P. and Queeny, Free convection Heat and Mass Transfer along a Vertical Surface in a Porous Media; Acta Mechanica, 123, pp.69-73, 1997.

[5] Nield, D. A. and Bejan, A., Convection in Porous Media, second edition, Springer-Verlag, New York, 1999. 